\title{
Predictors of Daily and Occasional Smoking and Quitting in Irish University Students
}

\author{
Gabrielle McKee ${ }^{*}$, Joe Barry², Martina Mullin³, Shane Allwright ${ }^{2}$, Catherine Hayes ${ }^{2}$ \\ ${ }^{1}$ School of Nursing and Midwifery, Institute of Population Health, Trinity College Dublin, The University of Dublin, Dublin, \\ Ireland \\ ${ }^{2}$ Public Health \& Primary Care, Institute of Population Health, School of Medicine, Trinity College Dublin, The University of \\ Dublin, Dublin, Ireland \\ ${ }^{3}$ College Health Centre, Trinity College Dublin, The University of Dublin, Dublin, Ireland \\ Email: *gmckee@tcd.ie
}

How to cite this paper: McKee, G., Barry, J., Mullin, M., Allwright, S. and Hayes, C. (2017) Predictors of Daily and Occasional Smoking and Quitting in Irish University Students. Health, 9, 435-450.

https://doi.org/10.4236/health.2017.93031

Received: February 1, 2017

Accepted: March 7, 2017

Published: March 10, 2017

Copyright (c) 2017 by authors and Scientific Research Publishing Inc. This work is licensed under the Creative Commons Attribution International License (CC BY 4.0).

http://creativecommons.org/licenses/by/4.0/

\begin{abstract}
Knowledge of student smoking behaviors and attitudes was sought to inform a proposal to implement a smoke-free university campus. This study assessed prevalence of student smoking and quitting behaviors and associated demographic, contextual and attitudinal factors. A cross-sectional design using a convenience sample on a single university site was utilized. Data were collected by anonymous self-administered questionnaires and analyzed using bivariate and multivariable logistic models comparing smokers and non-smokers (Model 1) and daily with occasional smokers Model 2. Of 739 respondents, 19\% of students smoked; $12 \%$ occasional (non-daily) and 7\% daily smokers. Smokers were more likely to be $\geq 22$ years and to allow smoking inside their accommodation (Model 1). They were less likely to agree that tobacco was addictive and to consider those who smoked at parties and weekends to be regular smokers. In Model 2, daily smokers were older and nine times more likely to report increased difficulty in handling stress if they quit smoking. Forty-four percent of all smokers had attempted to quit in the past year, $35 \%$ of smokers intended to quit in the next 30 days and 53\% in the next six months. Forty percent did not use quitting supports. Occasional rather than daily smoking was more prevalent in university students. Smokers differed from non-smokers in their perception of smoking-related health risks. Increasing age was the best predictor of regular and occasional smoking. Intentions to quit smoking and quit attempts were high among all smokers although use of on-site cessation supports was less than the optimal. These combined factors indicate a need for better targeting of our prevention strategies and the need to promote greater awareness of cessation supports for successful implementation of a smoke-free campus.
\end{abstract}

\section{Keywords}

Daily Smoking, Occasional Smoking, Smoking Cessation, University Student 


\section{Introduction}

University is a time of transition for many aspects of life in young adults, including health smoking behaviors [1]. It has been suggested that this is therefore, a population that may be receptive to health promotion initiatives, including smoking cessation [1] [2]. The background to this study was a proposal to introduce a tobacco free campus in a university in the Republic of Ireland. It was considered important to explore smoking and quitting behaviors and the attitudinal factors associated with these behaviors so as to better inform decision making on establishing a tobacco free campus.

A prior online survey of staff and student attitudes towards implementation of a tobacco free campus showed that the majority of the university community was in favor of the campus becoming tobacco-free (50\% of undergraduates, $58 \%$ of postgraduates and $66 \%$ of staff)

[https://www.tcd.ie/collegehealth/assets/documents/TFT\%20April\%202013\%20S urvey.pdf].

Smoking in young adults varies widely $(8 \%-34 \%)$ and the proportion of university students that smoke tend to be less than in their peers [3] [4]. The proportion of daily smokers ranges from $2 \%-16 \%$ and occasional (non-daily) smokers from $6 \%-12 \%$ [2] [3] [5] [6] [7]. A number of studies have examined the association between the student profile and social and cultural predisposing factors to smoking. Student smoking has been associated with many factors, including older age [8] [9] and non-health courses of study [4] [6] [9]. While craving is probably the strongest precipitator of smoking [10], situational variables are also important. Research has also found a significant association between smoking at home and current smoking status [11]. Studies that have compared predisposing factors to smoking in daily and occasional smokers have found that as occasional smokers more often define their smoking as social smoking [12], situational and contextual variables have been found to be stronger precipitators of smoking in occasional rather than daily smokers [10]. Contextual precipitators of smoking in occasional smokers include: location-where smoking is permitted such as outside [10]; presence of others smoking [10] [13]; alcohol [13] [14]; and stress [13].

Attitudes and beliefs about smoking risk differ according to smoking status [6]. Non-smokers are more likely to report exposure to smoking than smokers [15], have stronger beliefs with regard to second-hand smoke [15] and have higher perceived health-related risks from smoking [6]. Interestingly, occasional smokers, while they acknowledge smoking relates to health risks, minimize them as being personally relevant [12]. Knowledge and risk perception are important awareness factors that can influence motivation and therefore, mediate intention status with regard to smoking and quitting [16].

Overall approximately $50 \%$ of student smokers reported attempting to quit at some time or in the previous year [12] [14] [17] and, in general, occasional smokers were more likely to attempt to quit than daily smokers [12] [17]. The number of smokers intending to quit in the future varied from $9 \%-70 \%$, depend- 
ing on the type of smoker and the time frame of the question being asked e.g. quit in next year, next month [6] [12]. Berg et al., also found that occasional smokers had higher quit intentions than regular smokers and were more confident and motivated to quit; however, they were less likely to use behavioral interventions or pharmacotherapy [12] [17]. Female students, older students and those who had decreased the amount they smoked since attending university were more likely to want to quit and also more likely to plan to quit before graduation [8]. The university course attended did not have a significant association with students' intentions to stop smoking in the future [6]. Smoking fewer cigarettes a day, being a social smoker, having fewer friends who smoke and less frequent binge drinking were common additional factors associated with readiness to quit [12]. Intention to quit smoking was also related to the respondents' fear of the health-related effects of smoking, boredom factors, lack of self-confidence and cost [6] [12].

Previous experience with smoking bans has shown greater success in reducing second-hand smoking and its health effects than in reducing smoking itself [5] [18] [19]. Smoking bans have been linked to lower smoking prevalence and number of cigarettes smoked; however, this has not necessarily always translated into higher quit rates [20] [21] [22] [23]. Campus smoking bans are much more prevalent in America than in Europe and student attitudes to them are positive, particularly in the non-smokers [5] [20] [21].

The aim of this study was to assess prevalence of student smoking and quitting behaviors, and to identify the demographic, contextual and attitudinal factors associated with these behaviors as part of a move towards establishing a tobacco free university campus.

\section{Methods}

The study was a one site cross-sectional, descriptive study.

\subsection{Data Collection Tool}

A previously developed questionnaire meeting the aims of this study was not identified from review of the literature, therefore the study team developed a questionnaire utilizing, where possible, definitions and questions used in other studies. A pilot questionnaire was circulated for content validity for review by academic colleagues. The reviewers were asked to rate the questions for clarity and relevance on a four-part Likert scale, from very clear to not clear and from very relevant to not relevant. Seven questions were classified as "only somewhat clear" or "not clear" and seven were classified as "only somewhat relevant" or "not relevant". The research team then altered or deleted questions as appropriate based on these ratings and additional comments made by the reviewers. The final anonymous questionnaire included socio-demographic characteristics, exposure to secondhand smoke and accommodation smoking rules, participants' beliefs about smoking and risk perceptions. Participants were asked to define their smoking status as non-smoker, daily smoker or non-daily/occasional smoker. Smokers were asked 
type of cigarettes usually smoked, number of cigarettes smoked and their smoking patterns on campus. Questions relating to quitting behaviors asked "During the past 12 months have you stopped smoking for 24 hours or longer because you were trying to quit smoking", supports used for quitting, and intention to quit in the next 30 days and next 6 months. The final question explored smokers' views on perceived benefits of giving up smoking.

\subsection{Sample and Recruitment}

Based on a population of approximately 7300 undergraduate students it was estimated that a sample of 290 was required to determine smoking prevalence [24]. Convenience sampling was the method used to achieve this target. First year and third year students in three schools within the university were selected (School of Nursing and Midwifery, School of Biochemistry and Immunology, and School of Psychology), this approximated to 1000 students. The inclusion criteria for the study were: students aged 18 years or older; students from the above three schools and students attending class on the day of the survey. Ethics approval was received from the Royal College of Physicians of Ireland Research Ethics Committee.

\subsection{Data Collection}

Access to the students was arranged through the relevant School coordinators. Data collection occurred in the classroom. Module lecturers facilitated access to students during 15 minutes of class time; questionnaires were distributed and collected by members of the research team or administration personnel. To encourage participation, all students who submitted a questionnaire were invited to submit a detachable separate slip with email address for entry into a draw for a tablet computer. Questionnaires were administered to six classes; over a two-week period in October 2013.

\subsection{Data Analysis}

Data were analyzed using SPSS version 22. Descriptive statistics were used to describe the population. Bivariate analyses were conducted using Chi squared tests or Fisher's exact test as appropriate to examine associations between non-smokers and smokers and occasional and daily smokers' profiles, perceptions, beliefs and behaviors. Significant variables in bivariate analyses were then examined using two logistic regression models (backward, stepwise): Model 1 comparing smokers and non-smokers, Model 2 comparing daily and occasional smokers. Significance level for entry into the model was 0.1 . The significance level for variable retention in the models and for bivariate analyses was set at 0.05 .

\section{Results}

The estimated population was 1000; as there were 750 respondents this represents an estimated response rate of $75 \%$. However, class attendance was not recorded; as a consequence, the exact response rate to the survey could not be calculated. Eleven respondents did not complete the question on smoking habits and were 
excluded from the analysis. Of the remaining 739 respondents, the majority were biochemistry students (55\%), first year students (70\%), and aged less than 22 (84\%). Most were non-smokers (81\%). Smoking was significantly more common among nursing and psychology students, in those aged 22 years or more, and in those living in their own home (Table 1).

There were significant differences in the behaviors and in the beliefs held by smokers and non-smokers. In bivariate analysis, non-smokers were more likely to report never being exposed to second-hand smoke indoors ( $81 \%$ vs. $57 \%$ ) but less likely to report never being affected by smoking outside buildings on campus ( $40 \%$ vs. $56 \%)$. Non-smokers were more likely to say that smoking was not allowed anywhere inside their accommodation (88\% vs. 67\%). Smokers were significantly less likely than non-smokers to agree that people risk harming themselves by smoking only at weekends or on a few days per week (67\% vs. $80 \%$ ), to consider those who smoke at parties and weekends to be regular smokers $(21 \%$ vs. $48 \%$ ) or to agree that tobacco is addictive ( $54 \%$ vs. $74 \%$ ). Compared to non-smokers, smokers were more likely to report that they regarded the university's supports for quitting as being just right (26\% versus $18 \%$ ). However, over $60 \%$ of both groups said they were unsure if the supports were too little or just right (Table 2).

Model 1 examined the factors and beliefs which best predicted the odds of being a smoker or non-smoker. The final model, which contained four variables (age, accommodation rules, perception of smoking being addictive, perception

Table 1. Demographic comparison of smokers and non-smokers and bivariate analysis.

\begin{tabular}{|c|c|c|c|c|c|}
\hline $\begin{array}{l}\text { Variable } \\
\text { (n) }\end{array}$ & & $\begin{array}{c}\text { Total } \\
\mathrm{N}=739 \\
(100 \%)\end{array}$ & $\begin{array}{c}\text { Non-smokers } \\
\mathrm{N}=597 \\
(80.8 \%)\end{array}$ & $\begin{array}{l}\text { Smokers } \\
\mathrm{N}=142 \\
(19.2 \%)\end{array}$ & $\begin{array}{c}\chi^{2}(\mathrm{df}) \\
\mathrm{p}\end{array}$ \\
\hline & & n (\%) & n (\%) & $\mathrm{n}(\%)$ & \multirow{4}{*}{$\begin{array}{c}7.45(2) \\
0.03^{*}\end{array}$} \\
\hline \multirow{3}{*}{$\begin{array}{c}\text { School } \\
(738)\end{array}$} & Biochemistry & $408(55.3)$ & $344(84.3)$ & $64(15.7)$ & \\
\hline & $\begin{array}{l}\text { Nursing and } \\
\text { Midwifery }\end{array}$ & $260(35.2)$ & $199(76.5)$ & $61(23.5)$ & \\
\hline & Psychology & $70(9.5)$ & $53(75.7)$ & $17(24.3)$ & \\
\hline \multirow{2}{*}{$\begin{array}{l}\text { Age } \\
(739)\end{array}$} & $<22$ & $622(84.2)$ & $518(83.3)$ & $104(16.7)$ & \multirow{2}{*}{$\begin{array}{c}14.76(1) \\
<0.01^{\star}\end{array}$} \\
\hline & $\geq 22$ & $117(15.8)$ & $79(67.5)$ & $38(32.5)$ & \\
\hline \multirow{2}{*}{$\begin{array}{l}\text { Gender } \\
(446)\end{array}$} & Male & $153(34.3)$ & $121(79.1)$ & $32(20.9)$ & \multirow{2}{*}{$\begin{array}{c}1.32(1) \\
0.25\end{array}$} \\
\hline & Female & $293(65.7)$ & $246(84.0)$ & $47(16.0)$ & \\
\hline \multirow{3}{*}{$\begin{array}{c}\text { Current year } \\
(738)\end{array}$} & First year & $514(69.6)$ & $417(81.1)$ & $97(18.9)$ & \multirow{3}{*}{$\begin{array}{c}0.08(1) \\
0.78\end{array}$} \\
\hline & Third Year & $224(30.4)$ & $179(79.9)$ & $45(20.1)$ & \\
\hline & Living with parents & $394(53.3)$ & $329(83.5)$ & $65(16.5)$ & \\
\hline \multirow{3}{*}{$\begin{array}{c}\text { Main living } \\
\text { accommodation } \\
\text { (739) }\end{array}$} & Living in own home & $57(7.7)$ & $38(66.7)$ & $19(33.3)$ & \multirow{3}{*}{$\begin{array}{c}9.52(3) \\
0.03^{*}\end{array}$} \\
\hline & Living in college & $121(16.4)$ & $98(81)$ & $23(19)$ & \\
\hline & $\begin{array}{l}\text { Other e.g. rented } \\
\text { rooms or flats }\end{array}$ & $167(22.6)$ & $132(79)$ & $35(21)$ & \\
\hline
\end{tabular}

${ }^{*} \mathrm{p}<0.05$. 
Table 2. Bivariate comparison of beliefs, attitudes and behaviors of smokers and nonsmokers.

\begin{tabular}{|c|c|c|c|c|}
\hline Variable (n) & & $\begin{array}{l}\text { Non-smokers } \\
\qquad \begin{array}{l}\mathrm{N}=597 \\
(80.8 \%)\end{array}\end{array}$ & $\begin{array}{l}\text { Smokers } \\
\mathrm{N}=142 \\
(19.2 \%)\end{array}$ & $\begin{array}{l}\chi^{2}(\mathrm{df}) \\
\mathrm{p}\end{array}$ \\
\hline & & $\begin{array}{c}\mathrm{n} \\
(\%)\end{array}$ & $\begin{array}{c}\mathrm{n} \\
(\%)\end{array}$ & \\
\hline $\begin{array}{l}\text { Exposed to tobacco smoke } \\
\text { indoors from others smoking } \\
\end{array}$ & Never & $\begin{array}{c}484 \\
(81.1)\end{array}$ & $\begin{array}{c}81 \\
(57)\end{array}$ & $\begin{array}{l}43.86(2) \\
<0.01^{*}\end{array}$ \\
\hline \multirow{4}{*}{$\begin{array}{l}\text { Are you affected by smoking } \\
\text { outside the buildings on } \\
\text { campus that you use }(548)\end{array}$} & $<1$ hour day & $\begin{array}{c}88 \\
(14.7)\end{array}$ & $\begin{array}{c}38 \\
(26.8)\end{array}$ & \multirow{4}{*}{$\begin{array}{l}9.83(2) \\
<0.01^{*}\end{array}$} \\
\hline & $\geq 1$ hour day & $\begin{array}{c}25 \\
(4.2)\end{array}$ & $\begin{array}{c}23 \\
(16.2)\end{array}$ & \\
\hline & Never & $\begin{array}{c}182 \\
(40.1)\end{array}$ & $\begin{array}{c}53 \\
(56.4)\end{array}$ & \\
\hline & Occasionally & $\begin{array}{c}223 \\
(49.1)\end{array}$ & $\begin{array}{c}37 \\
(39.4)\end{array}$ & \\
\hline \multirow{3}{*}{$\begin{array}{l}\text { Rules about smoking inside your } \\
\text { accommodation (701) }\end{array}$} & Often & $\begin{array}{c}49 \\
(10.8)\end{array}$ & $\begin{array}{c}4 \\
(4.3)\end{array}$ & \multirow{3}{*}{$\begin{array}{c}37.87(2) \\
<0.01^{\star}\end{array}$} \\
\hline & $\begin{array}{l}\text { Not anywhere } \\
\text { inside }\end{array}$ & $\begin{array}{c}496 \\
(88.3)\end{array}$ & $\begin{array}{c}93 \\
(66.9)\end{array}$ & \\
\hline & $\begin{array}{c}\text { Some places/at } \\
\text { some times }\end{array}$ & $\begin{array}{c}48 \\
(8.5)\end{array}$ & $\begin{array}{c}34 \\
(24.5)\end{array}$ & \\
\hline \multirow{3}{*}{$\begin{array}{c}\text { People risk harming themselves } \\
\text { if they smoke } 1-5 \text { cigarettes a } \\
\text { day (736) }\end{array}$} & Everywhere inside & $\begin{array}{c}18 \\
(3.2)\end{array}$ & $\begin{array}{c}12 \\
(8.6)\end{array}$ & \multirow{3}{*}{$\begin{array}{c}2.84(1) \\
0.09\end{array}$} \\
\hline & Agree & $\begin{array}{c}568 \\
(95.5)\end{array}$ & $\begin{array}{c}129 \\
(91.5)\end{array}$ & \\
\hline & Disagree/Unsure & $\begin{array}{c}27 \\
(4.5)\end{array}$ & $\begin{array}{c}12 \\
(8.5)\end{array}$ & \\
\hline $\begin{array}{c}\text { People risk harming themselves } \\
\text { if they only smoke on a } \\
\text { weekend/a few days per week } \\
\text { (732) }\end{array}$ & Agree & $\begin{array}{c}475 \\
(80.1)\end{array}$ & $\begin{array}{c}93 \\
(66.9)\end{array}$ & $\begin{array}{l}10.53(1) \\
<0.01^{*}\end{array}$ \\
\hline \multirow{3}{*}{$\begin{array}{l}\text { Do you consider people who } \\
\text { smoke at weekends or parties to } \\
\text { be regular smokers }(728)\end{array}$} & Disagree/unsure & $\begin{array}{c}118 \\
(19.9)\end{array}$ & $\begin{array}{c}46 \\
(33.1)\end{array}$ & \multirow{3}{*}{$\begin{array}{l}45.98(2) \\
<0.01^{*}\end{array}$} \\
\hline & Agree & $\begin{array}{l}283 \\
(48)\end{array}$ & $\begin{array}{c}29 \\
(20.9)\end{array}$ & \\
\hline & Disagree & $\begin{array}{c}219 \\
(37.2)\end{array}$ & $\begin{array}{c}95 \\
(68.3)\end{array}$ & \\
\hline \multirow{3}{*}{$\begin{array}{l}\text { People get addicted to using } \\
\text { tobacco like they get addicted to } \\
\text { using cocaine/heroin }(727)\end{array}$} & Unsure & $\begin{array}{c}87 \\
(14.8)\end{array}$ & $\begin{array}{c}15 \\
(10.8)\end{array}$ & \multirow{3}{*}{$\begin{array}{l}18.49(1) \\
<0.01^{\star}\end{array}$} \\
\hline & Agree & $\begin{array}{c}435 \\
(73.6)\end{array}$ & $\begin{array}{c}74 \\
(54.4)\end{array}$ & \\
\hline & Disagree/unsure & $\begin{array}{c}156 \\
(26.4)\end{array}$ & $\begin{array}{c}62 \\
(45.6)\end{array}$ & \\
\hline $\begin{array}{l}\text { In relation to college supports for } \\
\text { people to stop smoking are they } \\
\text { (723) }\end{array}$ & Too little & $\begin{array}{c}82 \\
(14.1)\end{array}$ & $\begin{array}{c}13 \\
(9.3)\end{array}$ & $\begin{array}{c}6.48(2) \\
0.04^{*}\end{array}$ \\
\hline
\end{tabular}

${ }^{*} \mathrm{p}<0.05$. 
of party/weekend smokers as smokers $)(\mathrm{N}=592)$, was significant $\left(\chi^{2}=97.76, \mathrm{df}\right.$ $=5, \mathrm{p}<0.01)$ and correctly classified $81.8 \%$ of cases. Compared to non-smokers, smokers were significantly less likely to be under 22 years $(\mathrm{OR}=0.24, \mathrm{p}<0.01)$, more likely to allow smoking inside their accommodation $(\mathrm{p}<0.01)$, less likely to agree that tobacco is addictive $(\mathrm{OR}=0.42, \mathrm{p}<0.01)$ and much less likely to consider those who smoke at parties and weekends to be regular smokers (OR = $0.22, \mathrm{p}<0.01$ ) (Table 3 ). $15 \%$ - $24 \%$ of the variability between all smokers versus non-smokers was explained by this set of variables.

Twelve percent of respondents (88) defined themselves as occasional smokers and $7 \%$ (54) as daily smokers, representing a total of $19 \%$ (142) of the total population. Bivariate analysis indicated that there were significantly higher proportions of daily smokers than occasional smokers studying nursing or psychology than biochemistry, among students aged 22 years or more and those living in their own home (Table 4). There also were significant differences in the beliefs, perceptions and behaviors of occasional and daily smokers. On bivariate analysis, occasional smokers were more likely to agree that people risk harming themselves from smoking 1 - 5 cigarettes a day $(\mathrm{p}=0.01)$, whereas a greater proportion of daily smokers considered that people who smoke only at weekends or parties to be regular smokers $(\mathrm{p}=0.03)$. Occasional smokers were significantly less likely than daily smokers to report having smoked in the past 30 days: between classes ( $\mathrm{p}<0.01)$, at meals $(\mathrm{p}<0.01)$ and while drinking alcohol $(\mathrm{p}<0.01)$ (Table 5).

Forty-four percent of all smokers (56/126) reported making a quit attempt (purposefully stopped smoking for 24 hours or longer) in the 12 months prior to the study ( $42 \%$ of occasional and $48 \%$ of daily smokers). A total of $53 \%(62 / 116)$ of smokers said they intended to quit in the next six months (52\% occasional, $56 \%$ daily) and $35 \%(41 / 116)$ said they intended to quit in the next 30 days (43\% of occasional and $26 \%$ of daily smokers). The quitting behavior and intentions of daily and occasional smokers were not significantly different. Just under $60 \%$ of smokers reported using any help during their last quit attempt (84/142), but only a few $(<2 \%)$ reported using nicotine replacement therapy, varenicline or bupropion, or quit apps. Daily smokers were more likely than occasional smokers $(70 \%$ vs. $18 \%$ ) to report more difficulty in handling stress in their lives if they gave up smoking $(\mathrm{p}<0.01)$ (Table 5$)$. Just over $60 \%$ of both daily and occasional smokers smoked manufactured cigarettes; just under $40 \%$ smoked hand rolled cigarettes and less than $1 \%$ smoked cigars. Daily smokers $(n=54)$ reported smoking an average of $10 \pm 5$ cigarettes per day (range 1 - 25) while two-thirds (67\%) of occasional smokers $(n=66)$ reported smoking $\geq 5$ cigarettes per week.

Model 2, which examined the factors and beliefs which best predicted the odds of being an occasional or daily smoker was also significant $\left(\chi^{2}(\mathrm{~N}=88), 36.3 \mathrm{df}=\right.$ $2, \mathrm{p}<0.01$ ) (Table 6). Compared to occasional smokers, daily smokers were significantly less likely to be under 22 years $(\mathrm{OR}=0.2, \mathrm{p}<0.01)$ and were nine times more likely to report that it would be harder to handle stress in their life if they gave up smoking $(\mathrm{OR}=8.8, \mathrm{p}<0.01)$. Between $34 \%$ and $45 \%$ of the variability between daily and occasional smokers was explained by this set of variables (Table 6). 
Table 3. Multivariate logistic regression of factors associated with odds of smoking versus non-smoking (Model 1).

\begin{tabular}{|c|c|c|c|c|c|}
\hline \multirow[t]{2}{*}{ Variable } & \multirow[b]{3}{*}{$\geq 22$ (reference) } & \multirow{2}{*}{$\begin{array}{c}\text { Odds ratio } \\
\text { Non-smokers } \\
\text { vs. smokers }\end{array}$} & \multicolumn{2}{|c|}{$\begin{array}{l}\text { 95\% Confidence } \\
\text { intervals }\end{array}$} & \multirow[t]{2}{*}{$\begin{array}{c}\mathrm{p} \\
\text { value }\end{array}$} \\
\hline & & & Lower & Upper & \\
\hline \multirow{2}{*}{ Age group (years) } & & 1.00 & & & \\
\hline & $<22$ & 0.24 & 0.14 & 0.43 & $<0.01^{*}$ \\
\hline \multirow{3}{*}{$\begin{array}{l}\text { Rules about smoking } \\
\text { inside accommodation }\end{array}$} & $\begin{array}{l}\text { Smoking not } \\
\text { allowed inside } \\
\text { (reference) }\end{array}$ & 1.00 & & & \\
\hline & $\begin{array}{l}\text { Smoking allowed } \\
\text { some places/times }\end{array}$ & 2.98 & 1.62 & 5.51 & $<0.01^{*}$ \\
\hline & $\begin{array}{l}\text { Smoking allowed } \\
\text { everywhere inside }\end{array}$ & 4.23 & 1.76 & 10.15 & $<0.01^{*}$ \\
\hline $\begin{array}{l}\text { People get addicted to } \\
\text { using tobacco like they }\end{array}$ & Disagree/unsure & 1.00 & & & \\
\hline $\begin{array}{l}\text { get addicted to using } \\
\text { cocaine/heroin }\end{array}$ & $\begin{array}{l}\text { (reference) } \\
\text { Agree }\end{array}$ & 0.42 & 0.26 & 0.67 & $<0.01^{*}$ \\
\hline $\begin{array}{l}\text { Consider people who } \\
\text { smoke at weekends or }\end{array}$ & No (reference) & 1.00 & & & \\
\hline $\begin{array}{l}\text { parties to be regular } \\
\text { smokers }\end{array}$ & Yes & 0.22 & 0.13 & 0.45 & $<0.01^{*}$ \\
\hline
\end{tabular}

Table 4. Demographic comparison of occasional and daily smokers and bivariate analysis.

\begin{tabular}{|c|c|c|c|c|c|}
\hline $\begin{array}{l}\text { Variable } \\
\text { (n) }\end{array}$ & & $\begin{array}{c}\text { All } \\
\text { smokers } \\
\mathrm{N}=142 \\
(100 \%)\end{array}$ & $\begin{array}{c}\text { Occasional } \\
\text { smokers } \\
\mathrm{N}=88 \\
(62.0 \%)\end{array}$ & $\begin{array}{c}\text { Daily } \\
\text { smokers } \\
\mathrm{N}=54 \\
(38 \%)\end{array}$ & $\begin{array}{c}\chi^{2}(\mathrm{df}) \\
\mathrm{p}\end{array}$ \\
\hline \multirow{4}{*}{$\begin{array}{c}\text { School } \\
(142)\end{array}$} & & $\begin{array}{c}\mathrm{n} \\
(\%)\end{array}$ & $\mathrm{n}(\%)$ & $\mathrm{n}(\%)$ & \multirow{4}{*}{$\begin{array}{c}6.86(2) \\
0.03^{\star}\end{array}$} \\
\hline & Biochemistry & $64(45.1)$ & $47(73.4)$ & $17(26.6)$ & \\
\hline & $\begin{array}{l}\text { Nursing and } \\
\text { Midwifery }\end{array}$ & $61(43.0)$ & $31(50.8)$ & $30(49.2)$ & \\
\hline & Psychology & $17(12.0)$ & $10(58.8)$ & $7(41.2)$ & \\
\hline \multirow{2}{*}{$\begin{array}{l}\text { Age } \\
(142)\end{array}$} & $<22$ & $104(73.2)$ & $77(74.0)$ & $27(26.0)$ & \multirow{2}{*}{$\begin{array}{l}22.14(1) \\
<0.01^{\star}\end{array}$} \\
\hline & $\geq 22$ & $38(36.8)$ & $11(28.9)$ & $27(71.1)$ & \\
\hline \multirow[t]{2}{*}{$\begin{array}{l}\text { Gender } \\
(79)\end{array}$} & Male & $32(40.5)$ & $19(59.4)$ & $13(40.6)$ & \multirow{2}{*}{$\begin{array}{c}0.31(1) \\
0.58\end{array}$} \\
\hline & Female & $47(59.5)$ & $32(68.1)$ & 15 (31.9) & \\
\hline \multirow{3}{*}{$\begin{array}{l}\text { Current year } \\
\text { (142) }\end{array}$} & First year & $97(68.3)$ & $60(61.9)$ & $37(38.1)$ & \multirow{2}{*}{$\begin{array}{c}0.00(1) \\
1.00\end{array}$} \\
\hline & Third Year & $45(31.7)$ & $28(62.2)$ & $17(37.8)$ & \\
\hline & $\begin{array}{l}\text { Living with } \\
\text { parents }\end{array}$ & $\begin{array}{c}65 \\
(45.8)\end{array}$ & $43(66.2)$ & $22(33.8)$ & \multirow{4}{*}{$\begin{array}{l}12.3(3) \\
<0.01^{\star}\end{array}$} \\
\hline \multirow{3}{*}{$\begin{array}{c}\text { Main living } \\
\text { accommodation } \\
(142)\end{array}$} & $\begin{array}{l}\text { Living in own } \\
\text { home }\end{array}$ & $19(13.4)$ & $5(26.3)$ & $14(73.7)$ & \\
\hline & Living in college & $23(16.2)$ & $17(73.9)$ & $6(26.1)$ & \\
\hline & $\begin{array}{c}\text { Other e.g. rented } \\
\text { rooms or flats }\end{array}$ & $35(24.6)$ & $23(65.7)$ & $12(34.3)$ & \\
\hline
\end{tabular}

${ }^{*} \mathrm{p}<0.5$. 
Table 5. Bivariate comparison of beliefs, attitudes and behaviours of occasional and daily smokers.

\begin{tabular}{|c|c|c|c|c|}
\hline Variable & & $\begin{array}{c}\text { Occasional } \\
\text { smokers } \\
\mathrm{N}=88\end{array}$ & $\begin{array}{c}\text { Daily } \\
\text { smokers } \\
\mathrm{N}=54\end{array}$ & $\begin{array}{l}\chi^{2}(\mathrm{df}) \\
\mathrm{p}\end{array}$ \\
\hline \multirow{3}{*}{$\begin{array}{l}\text { Exposed to tobacco smoke } \\
\text { indoors from others } \\
\text { smoking }(142)\end{array}$} & \multirow[b]{2}{*}{ Never } & $\begin{array}{c}\mathrm{n} \\
(\%)\end{array}$ & $\begin{array}{c}\mathrm{n} \\
(\%)\end{array}$ & \\
\hline & & $\begin{array}{c}51 \\
(58)\end{array}$ & $\begin{array}{c}30 \\
(55.6)\end{array}$ & $0.01(1)$ \\
\hline & Any & $\begin{array}{c}37 \\
(42)\end{array}$ & $\begin{array}{c}24 \\
(44.4)\end{array}$ & 0.92 \\
\hline \multirow{3}{*}{$\begin{array}{l}\text { Are you affected by smoking outside } \\
\text { the buildings on } \\
\text { campus that you use (94) }\end{array}$} & Never & $\begin{array}{c}33 \\
(50)\end{array}$ & $\begin{array}{c}20 \\
(71.4)\end{array}$ & \multirow{3}{*}{$\begin{array}{c}2.85(1) \\
0.09\end{array}$} \\
\hline & Occasionally/often & $\begin{array}{c}33 \\
(50)\end{array}$ & $\begin{array}{c}8 \\
(28.6)\end{array}$ & \\
\hline & $\begin{array}{c}\text { Not anywhere } \\
\text { inside }\end{array}$ & $\begin{array}{c}62 \\
(72.1)\end{array}$ & $\begin{array}{c}31 \\
(58.5)\end{array}$ & \\
\hline \multirow[t]{2}{*}{$\begin{array}{l}\text { Rules about smoking inside your } \\
\text { accommodation (139) }\end{array}$} & $\begin{array}{c}\text { Some places/at } \\
\text { some times }\end{array}$ & $\begin{array}{c}18 \\
(20.9)\end{array}$ & $\begin{array}{c}16 \\
(30.2)\end{array}$ & $\begin{array}{c}2.77(2) \\
0.25\end{array}$ \\
\hline & Everywhere inside & $\begin{array}{c}6 \\
(7)\end{array}$ & $\begin{array}{c}6 \\
(11.3)\end{array}$ & \\
\hline $\begin{array}{l}\text { People risk harming } \\
\text { themselves if they smoke } 1-5\end{array}$ & Agree & $\begin{array}{c}84 \\
(96.6)\end{array}$ & $\begin{array}{c}45 \\
(83.3)\end{array}$ & \multirow{2}{*}{$0.01^{\star \#}$} \\
\hline cigarettes a day (141) & Disagree/Unsure & $\begin{array}{c}3 \\
(3.4)\end{array}$ & $\begin{array}{c}9 \\
(16.7)\end{array}$ & \\
\hline \multirow{2}{*}{$\begin{array}{l}\text { People risk harming themselves if } \\
\text { they only smoke on a weekend/a few } \\
\text { days per week (139) }\end{array}$} & Agree & $\begin{array}{c}57 \\
(67.1)\end{array}$ & $\begin{array}{c}36 \\
(66.7)\end{array}$ & \multirow{2}{*}{$\begin{array}{c}0.00(1) \\
1.0\end{array}$} \\
\hline & Disagree/unsure & $\begin{array}{c}28 \\
(32.9)\end{array}$ & $\begin{array}{c}18 \\
(33.3)\end{array}$ & \\
\hline \multirow{2}{*}{$\begin{array}{l}\text { Do you consider people who smoke } \\
\text { at weekends or parties to be regular } \\
\text { smokers (139) }\end{array}$} & Agree & $\begin{array}{c}12 \\
(14.1)\end{array}$ & $\begin{array}{c}17 \\
(31.5)\end{array}$ & \multirow{2}{*}{$\begin{array}{c}5.02(1) \\
0.03^{*}\end{array}$} \\
\hline & Disagree/unsure & $\begin{array}{c}73 \\
(85.9)\end{array}$ & $\begin{array}{c}37 \\
(68.5)\end{array}$ & \\
\hline \multirow{2}{*}{$\begin{array}{l}\text { People get addicted to using tobacco } \\
\text { like they get addicted to using } \\
\text { cocaine/heroin (136) }\end{array}$} & Agree & $\begin{array}{c}45 \\
(54.2)\end{array}$ & $\begin{array}{c}29 \\
(54.7)\end{array}$ & \multirow{2}{*}{$\begin{array}{c}0.00(1) \\
1.0\end{array}$} \\
\hline & Disagree/unsure & $\begin{array}{c}38 \\
(45.8)\end{array}$ & $\begin{array}{c}24 \\
(45.3)\end{array}$ & \\
\hline \multirow{2}{*}{$\begin{array}{l}\text { Usual type of cigarettes smoked } \\
\qquad(142)\end{array}$} & $\begin{array}{c}\text { Manufactured } \\
\text { cigarettes }\end{array}$ & $\begin{array}{c}54 \\
(61.4)\end{array}$ & $\begin{array}{c}34 \\
(63)\end{array}$ & $0.00(1)$ \\
\hline & Other & $\begin{array}{c}34 \\
(38.6)\end{array}$ & $\begin{array}{c}20 \\
(37)\end{array}$ & 1.0 \\
\hline \multicolumn{5}{|l|}{$\begin{array}{l}\text { Over the past } 30 \text { days have you } \\
\text { smoked on campus: }\end{array}$} \\
\hline \multirow{2}{*}{$\begin{array}{l}\text { At short breaks between classes } \\
(142)\end{array}$} & Yes & $\begin{array}{c}9 \\
(10.2)\end{array}$ & $\begin{array}{c}39 \\
(72.2)\end{array}$ & \multirow{2}{*}{$\begin{array}{c}54.48 \\
(1) \\
<0.01^{\star}\end{array}$} \\
\hline & No & $\begin{array}{c}79 \\
(89.8)\end{array}$ & $\begin{array}{c}15 \\
(27.8)\end{array}$ & \\
\hline \multirow{4}{*}{$\begin{array}{l}\text { While drinking alcohol } \\
\qquad(142)\end{array}$} & Yes & $\begin{array}{c}9 \\
(10.2)\end{array}$ & $\begin{array}{c}37 \\
(68.5)\end{array}$ & \multirow{2}{*}{$\begin{array}{l}49.29 \\
\quad(1) \\
<0.01^{\star}\end{array}$} \\
\hline & No & $\begin{array}{c}79 \\
(89.8)\end{array}$ & $\begin{array}{c}17 \\
(31.5)\end{array}$ & \\
\hline & Yes & $\begin{array}{c}23 \\
(26.1)\end{array}$ & $\begin{array}{c}31 \\
(57.4)\end{array}$ & 12.59 \\
\hline & No & $\begin{array}{c}65 \\
(73.9)\end{array}$ & $\begin{array}{c}23 \\
(42.6)\end{array}$ & $<0.01^{*}$ \\
\hline \multirow{2}{*}{$\begin{array}{c}\text { At parties } \\
(142)\end{array}$} & Yes & $\begin{array}{c}31 \\
(35.2)\end{array}$ & $\begin{array}{c}28 \\
(51.9)\end{array}$ & $3.16(1)$ \\
\hline & No & $\begin{array}{c}57 \\
64.8\end{array}$ & $\begin{array}{c}26 \\
48.1\end{array}$ & 0.08 \\
\hline
\end{tabular}




\section{Continued}

\begin{tabular}{|c|c|c|c|c|}
\hline $\begin{array}{l}\text { During the past } 12 \text { months have you } \\
\text { stopped smoking for } 24 \text { hours or }\end{array}$ & Yes & $\begin{array}{c}31 \\
(41.9)\end{array}$ & $\begin{array}{c}25 \\
(48.1)\end{array}$ & \multirow{2}{*}{$\begin{array}{c}0.26(1) \\
0.61\end{array}$} \\
\hline $\begin{array}{c}\text { longer because you were trying to } \\
\text { quit smoking (126) }\end{array}$ & No & $\begin{array}{c}43 \\
(58.1)\end{array}$ & $\begin{array}{c}27 \\
(51.9)\end{array}$ & \\
\hline \multirow{2}{*}{$\begin{array}{l}\text { Do you intend to quit smoking in } \\
\text { next } 6 \text { months }(116)\end{array}$} & Yes & $\begin{array}{c}33 \\
(51.6)\end{array}$ & $\begin{array}{c}29 \\
(55.8)\end{array}$ & \multirow{2}{*}{$\begin{array}{c}0.07(1) \\
0.79\end{array}$} \\
\hline & No & $\begin{array}{c}31 \\
(48.4)\end{array}$ & $\begin{array}{c}23 \\
(44.2)\end{array}$ & \\
\hline \multirow{2}{*}{$\begin{array}{l}\text { Do you plan to quit in next } 30 \text { days } \\
\qquad(116)\end{array}$} & Yes & $\begin{array}{c}27 \\
(42.9)\end{array}$ & $\begin{array}{c}14 \\
(26.4)\end{array}$ & \multirow{2}{*}{$2.72(1)$} \\
\hline & No & $\begin{array}{c}36 \\
(57.1)\end{array}$ & $\begin{array}{c}39 \\
(73.6)\end{array}$ & \\
\hline \multirow{2}{*}{$\begin{array}{l}\text { During your last attempt to give up, } \\
\text { did you use any help? (142) }\end{array}$} & Yes & $\begin{array}{c}56 \\
(63.6)\end{array}$ & $\begin{array}{c}28 \\
(51.9)\end{array}$ & \multirow{2}{*}{$\begin{array}{c}1.47(1) \\
0.23\end{array}$} \\
\hline & No & $\begin{array}{c}32 \\
(36.4)\end{array}$ & $\begin{array}{c}26 \\
(48.1)\end{array}$ & \\
\hline \multirow{3}{*}{$\begin{array}{l}\text { College supports for people to stop } \\
\text { smoking are: }(140)\end{array}$} & Too little & $\begin{array}{c}8 \\
(9.2)\end{array}$ & $\begin{array}{c}5 \\
(9.4)\end{array}$ & \multirow{3}{*}{$\begin{array}{c}2.62(2) \\
0.27\end{array}$} \\
\hline & Just right & $\begin{array}{c}19 \\
(21.8)\end{array}$ & $\begin{array}{c}18 \\
(34)\end{array}$ & \\
\hline & Unsure & $\begin{array}{c}60 \\
(69)\end{array}$ & $\begin{array}{c}30 \\
(56.6)\end{array}$ & \\
\hline \multicolumn{5}{|l|}{$\begin{array}{l}\text { In your opinion if you gave up } \\
\text { smoking: }\end{array}$} \\
\hline \multirow{2}{*}{$\begin{array}{l}\text { Your health would improve in the } \\
\text { short term (105) }\end{array}$} & Yes & $\begin{array}{c}44 \\
(73.3)\end{array}$ & $\begin{array}{c}39 \\
(86.7)\end{array}$ & \multirow[t]{2}{*}{$\begin{array}{c}2.01(1) \\
0.16\end{array}$} \\
\hline & No/unsure & $\begin{array}{c}16 \\
(26.7)\end{array}$ & $\begin{array}{c}6 \\
(13.3)\end{array}$ & \\
\hline \multirow{2}{*}{$\begin{array}{l}\text { Your health would benefit in the } \\
\text { long term (112) }\end{array}$} & Yes & $\begin{array}{c}59 \\
(93.7)\end{array}$ & $\begin{array}{l}48 \\
(98)\end{array}$ & \multirow{2}{*}{$0.38^{\#}$} \\
\hline & No/unsure & $\begin{array}{c}4 \\
(6.3)\end{array}$ & $\begin{array}{c}1 \\
(2)\end{array}$ & \\
\hline \multirow{2}{*}{ You would put on weight (99) } & Yes & $\begin{array}{c}14 \\
(26.4)\end{array}$ & $\begin{array}{c}21 \\
(45.7)\end{array}$ & \multirow[t]{2}{*}{$\begin{array}{c}3.19(1) \\
0.07\end{array}$} \\
\hline & No/unsure & $\begin{array}{c}39 \\
(73.6)\end{array}$ & $\begin{array}{c}25 \\
(54.3)\end{array}$ & \\
\hline \multirow{2}{*}{$\begin{array}{l}\text { It would be harder to handle stress } \\
\text { in your life (88) }\end{array}$} & Yes & $\begin{array}{c}8 \\
(17.8)\end{array}$ & $\begin{array}{c}30 \\
(69.8)\end{array}$ & $\begin{array}{l}22.15 \\
(1)\end{array}$ \\
\hline & No/unsure & $\begin{array}{c}37 \\
(82.2)\end{array}$ & $\begin{array}{c}13 \\
(30.2)\end{array}$ & $<0.01^{\star}$ \\
\hline \multirow{2}{*}{$\begin{array}{l}\text { You would feel you had done } \\
\text { something worthwhile (91) }\end{array}$} & Yes & $\begin{array}{c}39 \\
(79.6)\end{array}$ & $\begin{array}{c}38 \\
(90.5)\end{array}$ & \multirow[t]{2}{*}{$\begin{array}{c}1.31(1) \\
0.25\end{array}$} \\
\hline & No/unsure & $\begin{array}{c}10 \\
(20.4)\end{array}$ & $\begin{array}{c}4 \\
(9.5)\end{array}$ & \\
\hline \multirow{2}{*}{ You would save money (107) } & Yes & $\begin{array}{c}48 \\
(87.3)\end{array}$ & $\begin{array}{c}49 \\
(94.2)\end{array}$ & \multirow{2}{*}{$0.32^{*}$} \\
\hline & No/unsure & $\begin{array}{c}7 \\
(12.7)\end{array}$ & $\begin{array}{c}3 \\
(5.8)\end{array}$ & \\
\hline
\end{tabular}

\#Fisher's exact test; ${ }^{*} \mathrm{p}<0.05$.

\section{Discussion}

This study provides a comprehensive analysis of student smoking and quitting behaviors, their attitudes and perceptions of smoking, comparing not only smokers and non-smokers, but also comparing daily with occasional smokers. The The proportion of students in this study who were smokers was $19 \%, 12 \%$ were occasional smokers. A substantial proportion of both occasional (42\%) and daily 
Table 6. Multivariate logistic regression of factors associated with odds of occasional versus daily smoking (Model 2).

\begin{tabular}{|c|c|c|c|c|c|}
\hline \multirow[t]{2}{*}{ Variable } & & Odds ratio & $\begin{array}{c}95 \% \\
\text { Confidence } \\
\text { intervals }\end{array}$ & $\begin{array}{c}\mathrm{p} \\
\text { value }\end{array}$ & \\
\hline & & $\begin{array}{c}\text { Daily vs. } \\
\text { occasional } \\
\text { smokers }\end{array}$ & $\begin{array}{l}\text { Lower } \\
\text { Upper }\end{array}$ & & \\
\hline Age group (years) & $\begin{array}{l}\quad \geq 22 \\
\text { (reference) } \\
\quad<22\end{array}$ & $\begin{array}{c}1.00 \\
0.2\end{array}$ & 0.06 & 0.64 & $<0.01^{\star}$ \\
\hline $\begin{array}{l}\text { People risk harming } \\
\text { themselves if they smoke } \\
1 \text { - } 5 \text { cigarettes a day }\end{array}$ & $\begin{array}{c}\text { Disagree/unsure } \\
\text { (reference) } \\
\text { Agree }\end{array}$ & $\begin{array}{l}1.00 \\
0.11\end{array}$ & 0.01 & 1.15 & 0.07 \\
\hline $\begin{array}{l}\text { If you gave up smoking } \\
\text { it would be harder to } \\
\text { handle stress in your life }\end{array}$ & $\begin{array}{c}\text { No (reference) } \\
\text { Yes }\end{array}$ & $\begin{array}{l}1.00 \\
8.76\end{array}$ & 2.99 & 25.69 & $<0.01^{\star}$ \\
\hline
\end{tabular}

smokers (48\%) had attempted unsuccessfully to quit in the last year and a high percentage of both occasional (36\%) and daily (48\%) smokers reported their most recent quit attempt being made without assistance. Increasing age was the single most important factor predicting smoking. Within the relatively narrow age range of this sample, daily smokers were more likely to be older than occasional smokers and of the opinion that giving up smoking would make it harder to handle stress in their lives.

The prevalence of smoking (19\%), is at the lower end of the range previously reported in university students and is in keeping with studies on young populations as a whole in Ireland (8\% - 44\%) [2]-[7] [9] [12] [14] [22] [25]. Although this is a sizable minority, it indicates that it is no longer the norm to smoke. Interestingly a large percentage of both non-smokers (60\%) and smokers (44\%) reported being affected by second-hand smoke outside the campus buildings. These factors may contribute to the de-normalization of smoking as a behavior on campus. However, care must be taken to support rather than alienate the smoking cohort with the introduction of campus smoking bans. Our findings support those of previous studies of university students: those who were younger were more likely to be non-smokers [8] [9]. However, in contrast to previous studies, multivariate analysis showed that smoking was not significantly related to school [3] [6] [26], or year of study [27]. These trends were also seen within smokers, where older age was the only profile variable associated with daily smoking in multivariate analysis. As in previous studies, the majority of student smokers considered themselves as occasional smokers [2] [13] [17], smoking 5 or fewer cigarettes per week, which can be categorized as very light smoking [28] [29]. Occasional smokers are often considered as those who are starting smoking or those who are attempting to quit [28]. Therefore, this is an ideal group to promote quitting, as they may be more receptive to health promotion and smoking cessation interventions [1] [2].

In line with previous student studies a high proportion of smokers in this study 
(58\% occasional, $52 \%$ daily) had attempted to quit smoking in the previous year [2] [5] [30]. In addition, a large proportion intended to quit in the next 6 months ( $52 \%$ occasional, $56 \%$ daily) or next 30 days ( $43 \%$ occasional, $26 \%$ daily). In contrast to other studies of young smokers, this cohort of university students did not show significant differences in intention to quit across the smoking categories [17] [30]. These figures identify a large gap between intention to quit and successful quitting. This indicates the need for a better understanding of motivations to quit and the need for the provision of appropriate quit supports to assist students in moving successfully from intention/contemplation to action.

This study identified several misconceptions and behaviors that could be addressed to help narrow this gap. As in previous research, the negative effects of smoking were recognized by all students. However, this alone was not enough to stimulate successful quitting [9]. In some aspects, smokers' risk perceptions were lower than those of non-smokers. Compared to non-smokers, fewer smokers perceived those who only smoke at weekend/parties to be regular smokers and that people get addicted to using tobacco like they get addicted to using heroin or cocaine [13] [29]. Occasional smoking as well as daily smoking has been shown to have significant negative impacts on health; this message needs to be communicated more strongly to occasional smokers [28]. As in previous research, a sizable proportion of students in this study did not use support in their quitting attempts [30]. The aforementioned misconception by almost half of smokers that smoking is not addictive may contribute to the underutilization of support mechanisms [31]. The high proportion of smokers classing themselves as occasional may indicate that these may be going through a transition phase-taking up smoking or quitting and therefore may be more open to smoking cessation interventions. This suggests that occasional smokers in particular should be addressed as a specific target group for smoking cessation; different treatment approaches may be needed and nicotine replacement may not be appropriate nor necessary for this group [30] [32] [33].

The less than optimal utilization of supports may in part be due to the high proportion of smokers who were unsure if the university had enough supports for students to quit smoking or the nature of those supports. This suggests that better advertising is required to highlight campus cessation supports available. Students' fears concerning difficulties in handling stress if they quit smoking indicates the need for supplementary interventions to help students cope with stress. It also indicates that the timing of smoking cessation media strategies not to coincide with exam periods may make them more likely to succeed.

Student exposure to second-hand smoke at home and in public places varies widely depending on country [33]. In this study, exposure to smoke indoors was low; reflecting home smoking rules and national policy, but high proportions of both smokers and non-smokers reported being affected by smoking outside campus buildings. Second-hand smoke as a key health promotion message has resulted in increased quitting in some non-daily smokers [34].

Continued sustained media campaigns have been shown to be effective in in- 
creasing quitting rates in occasional and daily smokers in the 20 - 30 age groups [35]. University cessation programs can offer the motivation to students to quit [36] by utilizing interventions such as quitting with a friend (buddy systems), confining referrals to a single center or by bringing education and cessation interventions to the student [37]. From our study, addressing misconceptions about health and harm of smoking may provide additional motivation to quit in the student population particularly among daily smokers. Smoking bans, including smoke-free campuses, have been linked to decreased prevalence of smoking and have been seen to provide limited impetus for quitting smoking [2] [5] [18] [19] [30]. To inform the development of an effective tobacco free campus policy, policy developers should be cognizant of the demographic characteristics and the smoking status of the population [15]. This paper has identified some of the known antecedents of smoking behavior which can be more beneficially incorporated into an integrated model of change and the supports needed to facilitate this change [16]. This needs to be addressed if we are to stop the transition to smoking that occurs in this age group: non-daily smokers have a higher risk of becoming continuous smokers than non-smokers with up to a third of non-daily smokers at age 21 becoming daily smokers by 26 [30]. Our findings will inform the development of content of smoking-related messages for on-site posters, information screens and social media and increase awareness of university cessation supports, which will aid the implementation of the smoke-free campus initiative.

A strength of the study was the high response rate. Collecting data separately from the lecturer may have facilitated participants in being honest in their replies. There were however a number of limitations. Content validation was not carried out in the target population. Class attendance was not recorded; as a consequence, the response rate to the survey could only be estimated. The responses of those who did not attend the class may potentially differ from attendees. Not all variables known to affect smoking in this cohort were recorded. Gender was not recorded in the nursing group. Smoking rate was self-reported, hence prevalence of smoking may be under-reported but the use of anonymous questionnaires will have mitigated this.

\section{Conclusion}

Nineteen percent of students smoked. Increasing age was the best predictor of regular and occasional smoking. Smokers differed from non-smokers in their perception of smoking-related health risks. Intention to quit smoking and quitting attempts were high among daily and occasional smokers. Current use of onsite cessation supports was less than the optimal. These combined factors indicate a need for greater targeting of our prevention strategies e.g. as part of the induction process for new students, and the need to promote greater awareness of the cessation supports available for students. It is expected that these targeted measures will result in greater usage of smoking cessation services, translating into higher quit rates and reversing the transition from occasional to regular smoking. 
Our findings will thus aid the implementation of a smoke-free campus.

\section{References}

[1] Ling, P.M. and Glantz, S.A. (2002) Why and How the Tobacco Industry Sells Cigarettes to Young Adults: Evidence from Industry Documents. American Journal of Public Health, 92, 908-916. https://doi.org/10.2105/AJPH.92.6.908

[2] Marsh, L., Robertson, L.A. and Cameron, C. (2014) Attitudes towards Smoke Free Campus Policies in New Zealand. New Zealand Medical Journal, 127, 87-98.

[3] Kypri, K. and Baxter, J. (2004) Smoking in a New Zealand University Student Sample. New Zealand Medical Journal, 117, U794.

[4] Lucenteforte, E., Vannacci, A., Cipollini, F., Gori, A., Santini, L., Franchi, G., et al. (2010) Smoking Habits among University Students in Florence: Is a Medical Degree Course the Right Choice? Preventive Medicine, 51, 429-430. https://doi.org/10.1016/j.ypmed.2010.08.009

[5] Almutairi, K.M. (2014) Attitudes of Students and Employees towards the Implementation of a Totally Smoke Free University Campus Policy at King Saud University in Saudi Arabia: A Cross Sectional Baseline Study on Smoking Behavior Following the Implementation of Policy. Journal of Community Health, 39, 894-900. https://doi.org/10.1007/s10900-014-9893-Z

[6] Haddad, L.G. and Malak, M.Z. (2002) Smoking Habits and Attitudes towards Smoking among University Students in Jordan. International Journal of Nursing Studies, 39, 793-802. https://doi.org/10.1016/S0020-7489(02)00016-0

[7] Howat, P., Hallett, J., Kypri, K., Maycock, B., Dhaliwal, S. and McManus, A. (2010) Tobacco Smoking in an Australian University Sample and Implications for Health Promotion. Preventive Medicine, 51, 425-426. https://doi.org/10.1016/j.ypmed.2010.08.015

[8] Harris, J.B., Schwartz, S.M. and Thompson, B. (2008) Characteristics Associated with Self-Identification as a Regular Smoker and Desire to Quit among College Students Who Smoke Cigarettes. Nicotine and Tobacco Research, 10, 69-76. https://doi.org/10.1080/14622200701704202

[9] Musmar, S.G. (2012) Smoking Habits and Attitudes among University Students in Palestine: A Cross-Sectional Study. Eastern Mediterranean Health Journal, 18, 454460.

[10] Cronk, N.J. and Piasecki, T.M. (2010) Contextual and Subjective Antecedents of Smoking in a College Student Sample. Nicotine and Tobacco Research, 12, $997-$ 1004. https://doi.org/10.1093/ntr/ntq136

[11] Cauchi, D. and Mamo, J. (2012) Smoking Health Professional Student: An Attitudinal Challenge for Health Promotion? International Journal of Environmental Research and Public Health, 9, 2550-2561. https://doi.org/10.3390/ijerph9072550

[12] Berg, C.J., Ling, P.M., Hayes, R.B., Berg, E., Nollen, N., Nehl, E., et al. (2012) Smoking Frequency among Current College Student Smokers: Distinguishing Characteristics and Factors Related to Readiness to Quit Smoking. Health Education Research, 27, 141-150. https://doi.org/10.1093/her/cyr106

[13] Brown, A.E., Carpenter, M.J. and Sutfin, E.L. (2011) Occasional Smoking in College: Who, What, When and Why? Addictive Behaviors, 36, 1199-1204. https://doi.org/10.1016/j.addbeh.2011.07.024

[14] El Ansari, W. and Stock, C. (2012) Factors Associated with Smoking, Quit Attempts and Attitudes towards Total Smoking Bans at University: A Survey of Seven Universities in England, Wales and Northern Ireland. Asian Pacific Journal of Cancer Pre- 
vention, 13, 705-714. https://doi.org/10.7314/APJCP.2012.13.2.705

[15] Williams, R.D., Barnes, J.T., Hunt, B.P. and Winborne, H. (2011) Health Beliefs Related to Secondhand Smoke and Smoke-Free Policies in a College Community. American Journal of Health Studies, 26, 152-159.

[16] De Vries, H., Mudde, A., Leijs, I., Charlton, A., Vartiainen, E., Buijs, G., et al. (2003) The European Smoking Prevention Framework Approach (EFSA): An Example of Integral Prevention. Health Education Research, 18, 611-626. https://doi.org/10.1093/her/cyg031

[17] Berg, C.J., Sutfin, E.L., Mendel, J. and Ahluwalia, J.S. (2012) Use of and Interest in Smoking Cessation Strategies among Daily and Nondaily College Student Smokers. Journal of American College Health, 60, 194-202. https://doi.org/10.1080/07448481.2011.586388

[18] Callinan, J.E., Clarke, A., Doherty, K. and Kelleher, C. (2010) Legislative Smoking Bans for Reducing Secondhand Smoke Exposure, Smoking Prevalence and Tobacco Consumption. Cochrane Database Systematic Reviews, CD005992.

[19] Frazer, K., Callinan, J.E., McHugh, J., van Baarsel, S., Clarke, A., Doherty, K., et al. (2016) Legislative Smoking Bans for Reducing Harms from Secondhand Smoke Exposure, Smoking Prevalence and Tobacco Consumption. Cochrane Database Systematic Reviews, CD005992.

[20] Lupton, J.R. and Townsend, J.L. (2015) A Systematic Review and Meta-Analysis of the Acceptability and Effectiveness of University Smoke-Free Policies. Journal of American College Health, 63, 238-247. https://doi.org/10.1080/07448481.2015.1015029

[21] Seo, D.C., Macy, J.T., Torabi, M.R. and Middlestadt, S.E. (2011) The Effect of a Smoke-Free Campus Policy on College Students' Smoking Behaviors and Attitudes. Preventive Medicine, 53, 347-352. https://doi.org/10.1016/j.ypmed.2011.07.015

[22] Ohmi, H., Okizaki, T., Meadows, M., Terayama, K. and Mochizuki, Y. (2013) An Exploratory Analysis of the Impact of a University Campus Smoking Ban on Staff and Student Smoking Habits in Japan. Tobacco Induced Diseases, 11, 11-19. https://doi.org/10.1186/1617-9625-11-19

[23] Wechsler, H., Lee, J.E. and Rigotti, N.A. (2001) Cigarette Use by College Students in Smoke-Free Housing: Results of a National Study. American Journal of Preventive Medicine, 20, 202-207. https://doi.org/10.1016/S0749-3797(00)00313-5

[24] Naing, L., Winn, T. and Rusli, B.N. (2006) Practical Issues in Calculating the Sample Size for Prevalence Studies. Archives of Orofacial Sciences, 1, 9-14.

[25] Sychareun, V., Hansana, V., Choummanivong, M., Nathavong, S., Chaleunvong, K. and Durham, J. (2013) Cross-Sectional Survey: Smoking among Medical, Pharmacy, Dental and Nursing Students, University of Health Sciences, Lao PDR. British Medical Journal Open, 3, e003042. https://doi.org/10.1136/bmjopen-2013-003042

[26] Sutfin, E.L., McCoy, T.P., Berg, C.J., Champion, H., Helme, D.W., O’Brien, M.C. and Wolfson, M. (2012) Tobacco Use by College Students: A Comparison of Daily and Nondaily Smokers. American Journal of Health Behavior, 36, 218-229. https://doi.org/10.5993/AJHB.36.2.7

[27] Ordas, B., Fernandez, D., Ordonez, C., Marques-Sanchez, P., Alvarez, M.J., Martinez, S., et al. (2015) Changes in Use, Knowledge, Beliefs and Attitudes Relating to Tobacco among Nursing and Physiotherapy Students: A 10-Year Analysis. Journal of Advanced Nursing, 71, 2326-2337. https://doi.org/10.1111/jan.12703

[28] Schane, R.E., Ling, P.M. and Glantz, S.A. (2010) Health Effects of Light and Intermittent Smoking: A Review. Circulation, 121, 1518-1522.

https://doi.org/10.1161/CIRCULATIONAHA.109.904235 
[29] Shiffman, S., Tindle, H., Li, X., Scholl, S., Dunbar, M. and Mitchell-Miland, C. (2012) Characteristics and Smoking Patterns of Intermittent Smokers. Experimental and Clinical Psychopharmacology, 20, 264-277. https://doi.org/10.1037/a0027546

[30] Robertson, L., Iosua, E., McGee, R. and Hancox, R.J. (2016) Nondaily, Low-Rate Daily and High-Rate Daily Smoking in Young Adults: A 17-Year Follow-Up. Nicotine \& Tobacco Research, 18, 943-949. https://doi.org/10.1093/ntr/ntv167

[31] Doucet, J.M., Velicer, W.F. and Laforge, R.G. (2007) Demographic Differences in Support for Smoking Policy Interventions. Addictive Behaviors, 32, 148-157. https://doi.org/10.1016/j.addbeh.2006.04.003

[32] Edwards, S.A., Bondy, S.J., Kowgier, M., McDonald, P.W. and Cohen, J.E. (2010) Are Occasional Smokers a Heterogeneous Group? An Exploratory Study. Nicotine \& Tobacco Research, 12, 1195-1202. https://doi.org/10.1093/ntr/ntq168

[33] Warren, C.W., Sinha, D.N., Lee, J., Lea, V. and Jones, N.R. (2009) Tobacco Use, Exposure to Secondhand Smoke and Training on Cessation Counseling among Nursing Students: Cross-Country Data from the Global Health Professions Student Survey (GHPSS), 2005-2009. The International Journal of Environmental Research and Public Health, 6, 2534-2549. https://doi.org/10.3390/ijerph6102534

[34] Schane, R.E., Prochaska, J.J. and Glantz, S.A. (2013) Counseling Nondaily Smokers about Secondhand Smoke as a Cessation Message: A Pilot Randomized Trial. Nicotine \& Tobacco Research, 15, 334-342. https://doi.org/10.1093/ntr/nts126

[35] Terry-McElrath, Y.M., Emery, S., Wakefield, M.A., O’Malle, P.M., Szczypka, G. and Johnston, L.D. (2013) Effects of Tobacco-Related Media Campaigns on Smoking among 20-30-Year-Old Adults: Longitudinal Data from the USA. Tobacco Control, 22, 38-45. https://doi.org/10.1136/tobaccocontrol-2011-050208

[36] Wechsler, H., Kelley, K., Seibring, M., Kuo, M. and Rigotti, N.A. (2001) College Smoking Policies and Smoking Cessation Programs: Results of a Survey of College Health Center Directors. Journal of American College Health, 49, 205-212. https://doi.org/10.1080/07448480109596305

[37] Mooney, D.K. (2001) Facilitating Student Use of Campus Smoking Cessation Services. Journal of American College Health, 50, 141-142.

https://doi.org/10.1080/07448480109596019 
Submit or recommend next manuscript to SCIRP and we will provide best service for you:

Accepting pre-submission inquiries through Email, Facebook, LinkedIn, Twitter, etc. A wide selection of journals (inclusive of 9 subjects, more than 200 journals)

Providing 24-hour high-quality service

User-friendly online submission system

Fair and swift peer-review system

Efficient typesetting and proofreading procedure

Display of the result of downloads and visits, as well as the number of cited articles Maximum dissemination of your research work

Submit your manuscript at: http://papersubmission.scirp.org/

Or contact health@scirp.org 